system would not be complete if it did not offer a solution to formally validate the knowledge obtained by each learner.

Two major problems seem to be opposed to such an assertion:

i) How to validate knowledge of each learner in fields where knowledge is quickly obsolete.

ii) How to evaluate knowledge acquired in a flexible way, without formal control on the identity of the user.

eBioMED.ch ${ }^{\circledast}$ is able to generate selfassessment sessions based on the objectives and the profile of each learner. The results of those evaluations are stored by the system and constitute the user profile. This profile changes according to the user's actions. It also changes, and this is specific to the $\boldsymbol{e B i o}$ MED.ch ${ }^{\odot}$ system, according to the evolution of knowledge in the fields concerned.

\section{Conclusions}

eBioMED.ch ${ }^{\odot}$ was developed to put the learner in the centre of the learning process. In addition we have built a system, which does not induce deep change in the organization of partner institutions. These goals would have not been reached without reforming the classical learning process and developing new navigation concepts (as prerequisites pathway link, personalized modules).

Future versions of $\boldsymbol{e B i o M E D . c h}{ }^{\odot}$ will provide tools allowing users to participate in a knowledge construction process.

Each learner will access a logbook containing users' documents and annotations. This information could be shared with other learners through the prerequisites/related topics network.

Received: February 20, 2003
[1] P.J. Magistretti, F. Holzer, M.R. Celio, B. Desvergne, A. Borbély, B. Zaerpour, 'Swiss Virtual Campus - eBiomed.ch Project', 2000.

[2] A.W. Bates, in 'Managing Technological Change: Strategies for College and University Leaders', Ed. Jossey-Bass, San Fransisco, 1999.

[3] P.J. Magistretti, B. Zaerpour, 'Opal Modules', Elsevier Science Publisher B.V., 1991.

\title{
Paul Rys zum 65sten
}

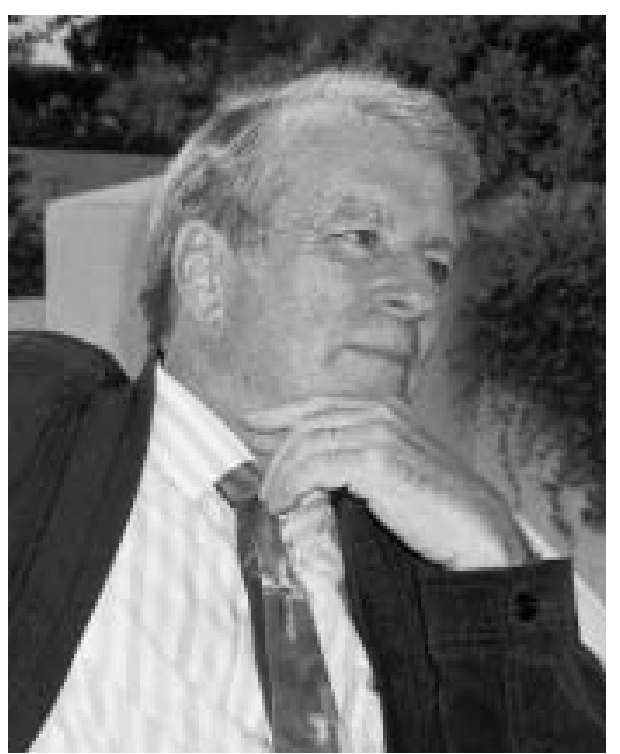

Am 3. April 2003 feiert Paul Rys seinen 65. Geburtstag. Mit herzlichen Glückwünschen blicken wir zurück auf 32 Jahre der Begeisterung für Forschung und Lehre im Departement Chemie, seit Anfang des Jahres Departement „Chemie und Angewandte Biowissenschaften" der ETH Zürich.

Paul Rys, 1938 in Prag geboren, kam 1946 in die Schweiz, verbrachte seine Jugend bis 1954 in Arosa (Graubünden) und schloss seine Schulbildung 1957 im Gymnasium Schwyz mit der Matura in naturwissenschaftlich-mathematischer Richtung ab. Nach dem Studium an der Abteilung für Chemie der ETH Zürich doktorierte Paul Rys 1965 bei Heinrich Zollinger mit einer Arbeit auf dem Gebiet der Reaktivfarbstoffe. Im Anschluss an zwei Forschungsaufenthalte an den Universitäten von Leeds und Manchester (1965/66) verbrachte er zwei Jahre bei Paul B. Weisz in den zentralen Forschungslaboratorien der Mobil Oil Corporation in Princeton. 1969 kehrte Paul
Rys in die Gruppe von Heinrich Zollinger an seiner alma mater zurück und wurde 1971 zum Assistenzprofessor für Farbstoffund Textilchemie ernannt. Es folgte 1975 die Wahl zum ausserordentlichen und 1984 jene zum ordentlichen Professor für Technische Chemie. In den Jahren 1982/83 und 1996/97 leitete Paul Rys als Vorsteher das Laboratorium für Technische Chemie, kürzlich umbenannt in „Institut für Chemie- und Bioingenieurwissenschaften".

Seine Doktorarbeit [1] und die beiden Jahre in der kreativen Forschungsatmosphäre bei Mobil Oil waren wegweisend für die weitere wissenschaftliche Arbeit von Paul Rys. Das Zusammenspiel von Transportprozessen, Adsorption und chemischer Reaktion [2] - bereits Thema seiner Habilitationsschrift „Über die Selektivität chemischer Prozesse“ (1971) - bildete den Ausgangspunkt für transdisziplinäre Forschung auf der Suche nach alternativen Synthesewegen für Feinchemikalien, sowie 
system would not be complete if it did not offer a solution to formally validate the knowledge obtained by each learner.

Two major problems seem to be opposed to such an assertion:

i) How to validate knowledge of each learner in fields where knowledge is quickly obsolete.

ii) How to evaluate knowledge acquired in a flexible way, without formal control on the identity of the user.

eBioMED.ch ${ }^{\circledast}$ is able to generate selfassessment sessions based on the objectives and the profile of each learner. The results of those evaluations are stored by the system and constitute the user profile. This profile changes according to the user's actions. It also changes, and this is specific to the $\boldsymbol{e B i o}$ MED.ch ${ }^{\odot}$ system, according to the evolution of knowledge in the fields concerned.

\section{Conclusions}

eBioMED.ch ${ }^{\odot}$ was developed to put the learner in the centre of the learning process. In addition we have built a system, which does not induce deep change in the organization of partner institutions. These goals would have not been reached without reforming the classical learning process and developing new navigation concepts (as prerequisites pathway link, personalized modules).

Future versions of $\boldsymbol{e B i o M E D . c h}{ }^{\odot}$ will provide tools allowing users to participate in a knowledge construction process.

Each learner will access a logbook containing users' documents and annotations. This information could be shared with other learners through the prerequisites/related topics network.

Received: February 20, 2003
[1] P.J. Magistretti, F. Holzer, M.R. Celio, B. Desvergne, A. Borbély, B. Zaerpour, 'Swiss Virtual Campus - eBiomed.ch Project', 2000.

[2] A.W. Bates, in 'Managing Technological Change: Strategies for College and University Leaders', Ed. Jossey-Bass, San Fransisco, 1999.

[3] P.J. Magistretti, B. Zaerpour, 'Opal Modules', Elsevier Science Publisher B.V., 1991.

\section{Paul Rys zum 65sten}

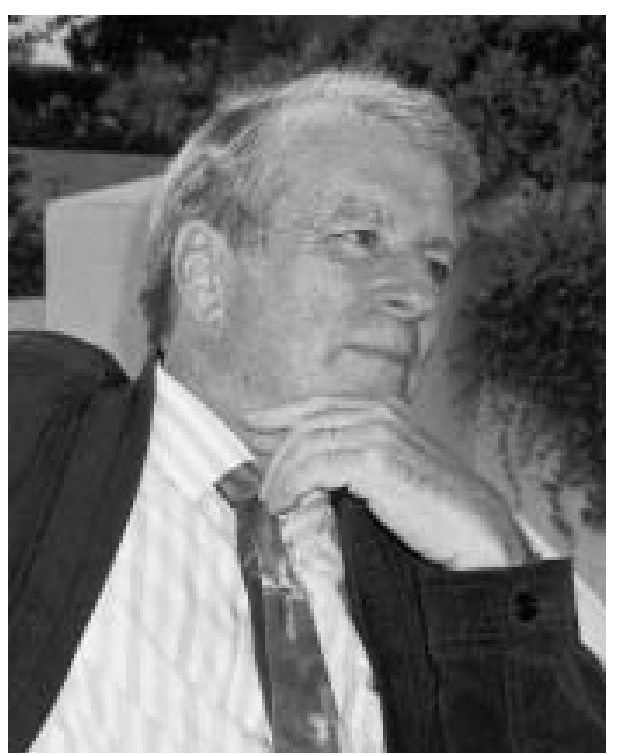

Am 3. April 2003 feiert Paul Rys seinen 65. Geburtstag. Mit herzlichen Glückwünschen blicken wir zurück auf 32 Jahre der Begeisterung für Forschung und Lehre im Departement Chemie, seit Anfang des Jahres Departement „Chemie und Angewandte Biowissenschaften" der ETH Zürich.

Paul Rys, 1938 in Prag geboren, kam 1946 in die Schweiz, verbrachte seine Jugend bis 1954 in Arosa (Graubünden) und schloss seine Schulbildung 1957 im Gymnasium Schwyz mit der Matura in naturwissenschaftlich-mathematischer Richtung ab. Nach dem Studium an der Abteilung für Chemie der ETH Zürich doktorierte Paul Rys 1965 bei Heinrich Zollinger mit einer Arbeit auf dem Gebiet der Reaktivfarbstoffe. Im Anschluss an zwei Forschungsaufenthalte an den Universitäten von Leeds und Manchester (1965/66) verbrachte er zwei Jahre bei Paul B. Weisz in den zentralen Forschungslaboratorien der Mobil Oil Corporation in Princeton. 1969 kehrte Paul
Rys in die Gruppe von Heinrich Zollinger an seiner alma mater zurück und wurde 1971 zum Assistenzprofessor für Farbstoffund Textilchemie ernannt. Es folgte 1975 die Wahl zum ausserordentlichen und 1984 jene zum ordentlichen Professor für Technische Chemie. In den Jahren 1982/83 und 1996/97 leitete Paul Rys als Vorsteher das Laboratorium für Technische Chemie, kürzlich umbenannt in „Institut für Chemie- und Bioingenieurwissenschaften".

Seine Doktorarbeit [1] und die beiden Jahre in der kreativen Forschungsatmosphäre bei Mobil Oil waren wegweisend für die weitere wissenschaftliche Arbeit von Paul Rys. Das Zusammenspiel von Transportprozessen, Adsorption und chemischer Reaktion [2] - bereits Thema seiner Habilitationsschrift „Über die Selektivität chemischer Prozesse“ (1971) - bildete den Ausgangspunkt für transdisziplinäre Forschung auf der Suche nach alternativen Synthesewegen für Feinchemikalien, sowie 
in der Farbstoff- und Textilchemie [3]. Mit Mitarbeiterinnen und Mitarbeitern aus sich jeweils ergänzenden Disziplinen führten auch Fragestellungen aus der Industrie immer zu grundlegenden Untersuchungen mathematischer, physikalisch-chemischer oder synthetischer Natur. Ein breites Spektrum von Themen ist gruppiert um die Selektivität chemischer Reaktionen und Prozesse. Als Beispiele seien genannt: die Kopplung Sorption/Diffusion [4], mischungsmaskierte Nitrierungen [5], Cyclometallierungen von Arylazo-Verbindungen [6], die Permeabilität von Membranen [7], ökonomisch und ökologisch vorbildliche Reduktionen von Nitrogruppen [8], oder das oszillierende Verhalten katalytischer Oxidationen [9]. Zahlreiche Untersuchungen über die Kopplung Stofftransport/chemische Reaktion mündeten schliesslich in die auch optisch eindrucksvolle Charakterisierung des Strömungsverhaltens chemischer Reaktoren mittels laserinduzierter Fluoreszenz-Tomographie mit hoher Zeitauflösung [10]. ,Nebenschauplätze' in Paul Rys' Forschung waren unter anderem die Synthese biologisch aktiver Moleküle [11] oder die elektrokatalytische Herstellung von Silizium-Folien [12]. In der Farbstoff- und Textilchemie standen zuletzt umwelttechnologisch nachhaltige Prozesse im Vordergrund, wie die elektrochemische [13] beziehungsweise elektrokatalytische [14] Reduktion von Küpenfarbstoffen, sowie die chemisch-oxidative und anaerob-mikrobielle Abwasserbehandlung [15].

Seine vielseitigen Forschungsinteressen kann Paul Rys mit grossem Talent verkaufen, im übertragenen und wörtlichen Sinn um einerseits seine Schülerinnen und Schüler zu begeistern, sowie um andererseits die heute besonders geforderten Drittmittel an die ETH zu bringen. Ende des Sommersemesters 2003 wird Paul Rys in den Ruhestand treten. Doch seine Liebe zu den Bündner Bergen, wie auch zu Südfrankreich, und sein Engagement in und für ETH-Spin-Offs werden ihn nicht zur Ruhe kommen lassen.

Lieber Paul, im Namen der ehemaligen und gegenwärtigen Mitarbeiterinnen und Mitarbeiter, der Kolleginnen und Kollegen, die herzlichsten Glückwünsche, sowie Freude und Erfolg für alle zukünftigen Aktivitäten!

Peter Skrabal

Institut für Chemie- und

Bioingenieurwissenschaften

ETH Hönggerberg, HCI

8093 Zürich
[1] 1. Publikation: P. Rys, Hch. Zollinger, 'Hydrolyse und Alkoholyse von Reaktivfarbstoffen: I. Hydrolyse von Dichlortriazin-Farbstoffen', Helv. Chim. Acta 1966, 49, 749-754.

[2] P. Rys, 'Disguised Chemical Selectivities', Acc. Chem. Res. 1976, 9, 345-351; 'Diffusionsmaskierte Selektivität chemischer Reaktionen. Teil I: Mischungsmaskierte chemische Selektivität', Angew. Chem. 1977, 89, 847-857, Angew. Chem. Int. Ed. Engl. 1977, 16, 807-817; 'Chemical Selectivities Disguised by Mixing', Pure Appl. Chem. 1981, 53, 209-221; 'The Mixing-Sensitive Product Distribution of Chemical Reactions', Chimia 1992, 46, 469-476.

[3] P. Rys, 'The Interaction of Mass Diffusion and Chemical Reaction During Finishing and Dyeing of Textiles', Text. Res. J. 1973, 43, 24-33.

[4] R.J. Ott, P. Rys, 'Sorption-Diffusion in Heterogeneous Systems. Part 8. - Appraisal of Diffusion Effects in Sorption Systems', J. Chem. Soc., Faraday Trans. I 1974, 70, 995-999.

[5] F. Nabholz, P. Rys, 'Chemical Selectivities Disguised by Mass Diffusion. IV. Mixing-Disguised Nitrations of Aromatic Compounds with Nitronium Salts, 4th Communication on the Selectivity of Chemical Processes', Helv. Chim. Acta 1977, 60, 2937-2943.

[6] A.J. Klaus, P. Rys, 'Cyclometalation of Arylazo Compounds. Part 1. Synthesis and Cyclopalladation of Some Substituted 1-Arylazonaphthalenes. 1st Communication on Compounds with a Metal-Arene $\sigma-$ Bond', Helv. Chim. Acta 1981, 64, 14521466.

[7] P. Rys, R. Weber, 'Diffusional Boundary Layers in a New Steady-State Diffusion Cell', J. Phys. Chem. 1990, 94, 83098315.

[8] M. Lauwiner, R. Roth, P. Rys, 'Reduction of aromatic nitro compounds with hydrazine hydrate in the presence of an iron oxide/hydroxide catalyst. III. The selective reduction of nitro groups in aromatic azo compounds', Appl. Catal., A 1999, 177, 9-14.

[9] P. Rys, J. Wang, 'Hydrogen Isotope Effect and Traveling Waves in a Halogen-Free Chemical Oscillatory System', J. Am. Chem. Soc. 1992, 114, 356-357.

[10] G.J. Merkel, P. Rys, F.S. Rys, T.A. Dracos, 'Concentration and Velocity Field Measurements in Turbulent Flows Using Laser-Induced Fluorescence Tomography (LIFT)', Appl. Sci. Res. 1996, 56, 181190.

[11] R. Baumann, P. Rys, 'Metachromatic activity of $\beta$-cyclodextrin sulfates as heparin mimics', Int. J. Biol. Macromol. 1999, 24 , 15-18.

[12] M. Dürrler, H.R. Grüniger, P. Rys, H. Kiess, W. Rehwald, 'Charakterisierung von elektrokatalytisch hergestellten Folien und Schichten aus Silizium', Ber. Bunsen-Ges. 1995, 99, 176-183.

[13] A. Roessler, O. Dossenbach, U. Meyer, W. Marte, P. Rys, 'Direct Electrochemical
Reduction of Indigo', Chimia 2001, 55, 879-882.

[14] A. Roessler, O. Dossenbach, P. Rys, 'Electrocatalytic Hydrogenation of Indigo. Process Optimization and Scale-Up in a Flow Cell', J. Electrochem. Soc. 2003, 150, D1-D5.

[15] M. Huber, U. Meyer, P. Rys, 'Biodegradation Mechanisms of Linear Alcohol Ethoxylates under Anaerobic Conditions', Environ. Sci. Technol. 2000, 34, 17371741. 